\title{
Models of 802.11 Multi-Hop Networks: Theoretical Insights and Experimental Validation
}

\author{
(Invited Paper) \\ Adel Aziz*, Mathilde Durvy ${ }^{\ddagger}$, Olivier Dousse ${ }^{\dagger}$, and Patrick Thiran* \\ ${ }^{*}$ School of Communications and Computer Sciences, EPFL, CH-1015 Lausanne, Switzerland \\ ${ }^{\dagger}$ Nokia Research Center, CH-1015 Lausanne, Switzerland \\ ${ }^{\ddagger}$ Cisco, CH-1180 Rolle, Switzerland
}

\begin{abstract}
Wireless Multi-Hop CSMA/CA Networks are challenging to analyze. On the one hand, their dynamics are complex and rather subtle effects may severely affect their performance. Yet, understanding these effects is critical to operate upper layer protocols, such as TCP/IP. On the other hand, their models tend to be very complex in order to reproduce all the features of the protocol. As a result, they do not convey much insight into the essential features.

We review two models of $\mathbf{8 0 2 . 1 1}$ protocols, which are simple enough to first explain why a trade-off needs to be found between fairness and spatial reuse (throughput) in saturated wireless networks (where all nodes have packets to transmit to their neighbors); and then to explain why non-saturated networks (where only some nodes, the sources, have packets to transmit to their destinations in a multi-hop fashion) that are more than 3 hops longs suffer from instability. We confront both models either to realistic simulations in ns-2 or to experiments with a testbed deployed at EPFL. We find that the predictions of both models help us understand the performance of the 802.11 protocol, and provide hints about the changes that need to be brought to the protocol.
\end{abstract}

\section{INTRODUCTION}

IEEE 802.11 is the most popular Medium Access Control (MAC) protocol in current wireless networks. In the singlehop wireless LAN setting, its performance is by now quite well understood. However, in the multi-hop setting where relay nodes are used to achieve end-to-end communication, there is, to date, no widely accepted model. Consequently, when confronted with experimental results, researchers and engineers often find it hard to interpret them.

We review two models of protocols "à la 802.11" in the context of wireless multi-hop ad hoc or mesh networks. They are simple enough to convey insight in certain key properties of these protocols in terms of throughput, fairness and stability. The details of the models described in this paper are found in $[1,2]$ and [3].

In both cases, we consider in general only a 1-dimensional, linear network topology, of $N+1$ nodes $\{0,1, \ldots, N\}$ placed on a line. The Receiver and Carrier Sensing Ranges are adjusted so that direct neighboring nodes can communicate with each other, but interfere with transmissions from any other neighbor of this pair of nodes. A direct link exists thus only between node $i$ and its neighboring node $i \pm 1$, for all $1 \leq i \leq N-1$. A link $(i, i+1)$ can be in two states, active $978-1-4244-8953-4 / 11 / \$ 26.00$ (C) 2011 IEEE (if there is a data transmission between $i$ and $i+1$ ) or idle. It can be active only when links $(i-1, i)$ and $(i+1, i+2)$ are idle. Although some of the findings described in the paper can be extended to other topologies and neigborhoods, linear topologies with nearest neighbor interference allow us to make explicit computations.

The first model described in Section II represents CSMA/CA networks where all nodes are saturated, that is, have a full queue of packets at all times. Every node has an infinite amount of data to send to every other node in the network. This setting is clearly extreme, but it allows us to focus on the long-range effects induced indirectly by the local coupling between Carrier Sensing and back-off mechanisms, without having to deal with traffic and routing matrices, queue states, packet arrival processes. Maximizing throughput boils down then to the maximization of the spatial reuse of the protocol, or, in other words, its ability to schedule a large number of concurrent successful transmissions. A fair allocation of resources implies that all nodes can access the wireless channel during an equal amount of time. In Section II-A, we will see that we can model such networks by the wellknown Markovian loss-network model [4], which provides rich insights into the unavoidable trade-offs between spatial reuse and fairness, described in Sections II-B and II-C. We will also comment about the differences between the model and the actual IEEE 802.11 protocols in Section II-D.

The second model of the paper, described in Section III, represents non-saturated CSMA networks on a linear topology, where one node only (the source, Node 0) is saturated, whereas all the other nodes (Nodes $1, \ldots, N-1$ ) only relay traffic generated by the source to the destination (Node $N$ ). In this situation, we again do not need to deal with traffic nor routing matrices, but we need to care about the queue states, which adds a considerable difficulty, compared to the loss model used for saturated network conditions. We will see however that a discrete-time Markov chain model, described in Sections III-A and III-B, can quite reliably reproduce the dynamics of the network, and, in particular, can explain why the network becomes unstable as soon as it has a length of 4 hops (Section III-C). We validate the model on a real testbed, and verify that the conclusions drawn from it hold for various data rates in Section III-D. 


\section{SATURATED NETWORK}

\section{A. Network model}

In this section, we work with an idealized version of the 802.11 protocol, where RTS/CTS messages are exchanged instantanesously. The model captures the essential features of the protocol, but leaves aside many effects of the real protocol - we will come back to them in Section II-D. This model, which is a loss network model [4], has already been used earlier in [5] as well as in [6].

We consider a line network composed of a sequence of $(N+1)$ equally spaced (by one space unit) nodes on a line where each pair of neighboring nodes is connected by a link. Because of the nearest neighbor intereference, and because of the RTS/CTS instantaneous exchange, both links $(i-1, i)$ and $(i+1, i+2)$ must be idle when link $(i, i+1)$ is active. We can therefore associate to each active link a domain, the exclusion domain, which is the portion of the line occupied by an active link and all the interfering links that must remain idle suring the transmission on the active link. In the line network (Figure 1), the exclusion domain of a link is a line segment of length 3. A transmission pattern on the line network is thus equivalent to a collection of non-overlapping line segments on the 1-dimensional lattice.

We assume that each link maintains a backoff timer, which is initialized to a random value chosen according to an exponential backoff distribution with mean $1 / \lambda$ (which corresponds to the average contention window size $c w$, see Section II-D). The timer of a link runs when the link sees the channel as idle; when the link senses the channel busy, it temporarily freezes its timer. A link becomes active when its backoff timer reaches zero, which is only possible if all the links in its exclusion set are idle. It stays active for an exponentially distributed time with mean $\mu^{-1}$. After each transmission, the link resets its backoff timer to a new random value. This mechanism slightly differs from the standard 802.11 protocol, where each node maintains a unique backoff timer for all its links.

We call transmission pattern the set of active links in the network at a given time (in other words, a transmission pattern is a an independent set of the contention graph of the network). A transmission pattern must respect the constraint specified by the exclusion domain of active links. For a given network, we say that a transmission pattern is maximal if there is no other transmission pattern with a larger number of active links (it is thus a maximal independent set of the contention graph of the network).

The dynamics of this idealized protocol can be described by a continuous time Markov chain, whose state space is the set of all possible transmission patterns in the network. This Markov chain has a unique stationary distribution given by

$$
\pi(x)=\frac{\rho^{n(x)}}{Z}
$$

where $x$ denotes a transmission pattern, $n(x)$ is the number of active links in $x, \rho=\lambda / \mu$ is the access intensity, and $Z=\sum_{x} \rho^{n(x)}$ is a normalizing constant. line segment of length 3

Fig. 1. Maximal transmission pattern on the line network, the exclusion domain of a link is a line segment of length 3 .

\section{B. Spatial Reuse}

The average spatial reuse $\sigma$ is defined as the number of simultaneously active links, normalized by the number $N$ of (non-directed, i.e. bi-directional) links in the network. It can be expressed, using (1), as

$$
\sigma=\frac{1}{N} \sum_{x} n(x) \pi(x)=\frac{1}{N} \frac{\sum_{x} n(x) \rho^{n(x)}}{\sum_{x} \rho^{n(x)}} .
$$

The spatial reuse measures the ability of the MAC protocol to schedule a large number of simultaneous transmissions. In the saturated network considered here, it is therefore directly proportional to the link throughput. Clearly, in the linear network with nearest neighbor interference, $\sigma \leq 1 / 3$ because each active consumes an exclusion domain of length 3 .

We computed in [6] the asymptotic value of (2) when $N \rightarrow$ $\infty$ (the same result can also be obtained from [7]). For the setting considered here, we find that as $N \rightarrow \infty$,

$$
\sigma \rightarrow \frac{2 \lambda y_{1}^{2}}{\mu+6 \lambda y_{1}^{2}}
$$

with $y_{1}$ the positive real root of $1-y-2(\lambda / \mu) y^{3}$.

Figure 2 shows the average spatial reuse as a function of $\lambda / \mu$, for a 50 node line network topology and when $N \rightarrow \infty$. As $\lambda / \mu$ increases (i.e., as the average backoff time becomes small compared to the average exchange time), the spatial reuse of the protocol reaches the maximal spatial reuse of 0.3469 (the value is slightly above $1 / 3$ because of the border effects). Consequently, when the average backoff time is much small than the average link activity duration, 802.11 (at least in its idealized version), is able to reach the maximal spatial reuse.

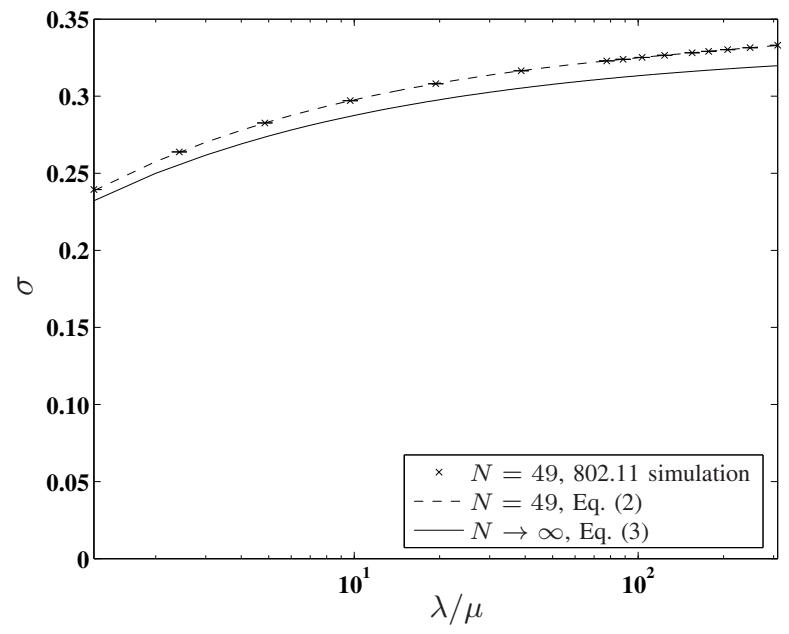

Fig. 2. Spatial reuse achieved by the idealized protocol on a line topology of 50 nodes, as a function of the access intensity $\lambda / \mu$. 


\section{Fairness}

To assess the MAC layer fairness of the protocol, we use Jain's Fairness Index [8]. Let

$$
p(i)=\sum_{x \mid x(i)=1} \pi(x)=\frac{\sum_{x \mid x(i)=1} \rho^{n(x)}}{\sum_{x} \rho^{n(x)}}
$$

be the probability that link $(i, i+1)$ on the line is active. The link fairness index of the protocol is

$$
\mathrm{FI}=\frac{\left(\sum_{i=0}^{N-1} p(i)\right)^{2}}{N \sum_{i=0}^{N-1} p^{2}(i)} .
$$

It reaches its maximal value, 1 , when all links access the channel equally.

The unfairness of 802.11 is due to the topological advantage of the links at the border of the network, over the ones in the center: the former compete with fewer links than the latter, and can therefore access the channel more often, this is often refer to as the "Flow in the Middle" problem (see e.g. [9, 10]). The ratio $\lambda / \mu$ indicates how far this effect propagates in the network, as shown in Figure 3. For finite values of $\lambda / \mu$, it will always stop at some point in the network, if $N$ is large enough. As a result, for large 1-dimensional linear networks, the protocol will be long term fair for nodes that sufficiently far away from the border, as shown on Figure 4. This is in sharp contrast with 2-dimensional networks, where the idealized 802.11 protocol may be unfair independently of the size of the network for large access intensities $\lambda / \mu$, because of a phase transition [2].

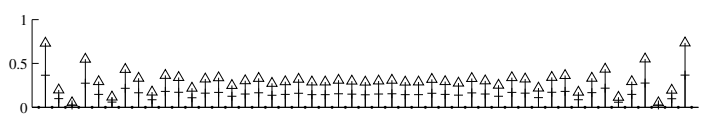

(a) $\lambda / \mu=20, \sigma=0.31, \mathrm{FI}=0.85$

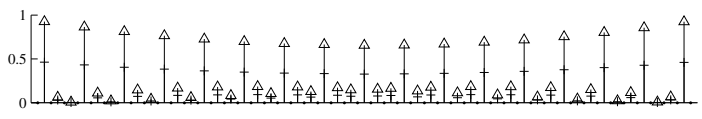

(b) $\lambda / \mu=620, \sigma=0.34$, FI $=0.54$

Fig. 3. Border effect on a 50 node line network, for different values of the access intensity $\lambda / \mu$. The line network is represented on the horizontal axis. We observe that as $\lambda / \mu$ increases, the border effect is felt deeper inside the network, and the protocol becomes less fair.

\section{Differences with the IEEE 802.11 Network Model}

We consider the implementation of IEEE 802.11 under the widely used ns-2 simulator [11]. The main reason for choosing this specific implementation is twofold: (i) The code is available, contrary to the code contained in the firmware of IEEE 802.11 cards on the market and (ii) it follows quite closely (but not completely) the IEEE 802.11 standard. The main limitation of the ns-2 simulator is at the physical layer: It does not support cumulative interference.

We use the default ns-2 parameters, which mimic a $914 \mathrm{MHz}$ Lucent WaveLAN DSSS radio interface. The RXRange and

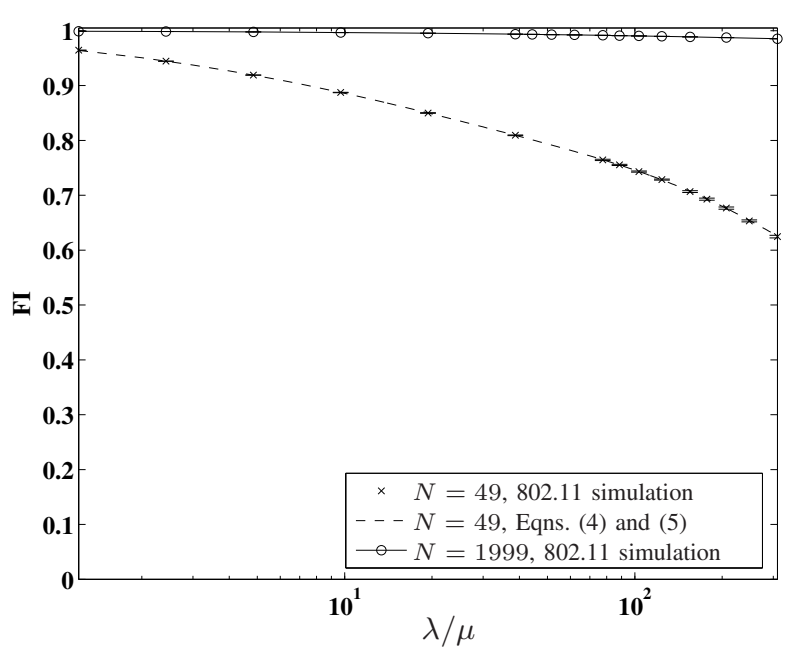

Fig. 4. Jain's Fairness Index (FI) computed at the end of a very long simulation of the idealized 802.11 protocol on a 50 and a 2000 node line network. The Jain Fairness Index is plotted as a function of the access intensity $\lambda / \mu$. The agreement between the simulation results and the values given by Equations (5), (4) is excellent.

CSRanges are $250 \mathrm{~m}$ and the capture threshold is equal to 10 , which means that a signal can capture the channel if it is 10 times stronger than the interfering signal.

The DATA packet size is 1500 bytes (i.e., the average exchange time $\mu^{-1}$ is fixed to a value slightly larger than $1500 \cdot 8 \cdot 10^{-6}$ ). To provide accurate results, each simulation is repeated 50 times (using different random seeds). The simulations run for 40 seconds of real time, after a warm-up period of 10 seconds. Figures 5 and 6 follow the same format. We plot the average spatial reuse $\sigma$ of the protocol as a function of its contention window $c w$ (i.e., as a function of the average backoff time $\left.\lambda^{-1}=0.5 \cdot c w \cdot 20 \cdot 10^{-6}\right)$. We consider fixed contention window cases (i.e., $c w=C W_{\min }=C W_{\max }$ ) and a variable contention window case (i.e., $C W_{\min } \leq c w \leq C W_{\max }$ ) where the contention window is doubled after a collision.

We compute the performance of the idealized 802.11 protocol model of Section II-A on a line topology of 50 nodes $^{1}$. We then simulate IEEE 802.11 using the ns-2 simulator on the same topology.

Figure 5 shows the performance of the idealized protocol together with the performance of (ns-2) IEEE 802.11. The average spatial reuse $\sigma$ of the idealized protocol increases towards the maximal value of 0.3469 as the contention window $c w$ becomes small. This is however not the case for the current IEEE 802.11 protocol. It achieves a spatial reuse of at most 0.17 , half the optimal spatial reuse of 0.3469 . Yet, we have seen that the high spatial reuse of the idealized protocol comes at the cost of a reduced fairness. In contrast, the current IEEE 802.11 protocol is relatively fair even at low $c w$ values. Its fairness index $(\mathrm{FI})$ oscillates between $0.80(\mathrm{cw}=3)$ and 0.95

\footnotetext{
${ }^{1}$ In the variable contention window case, the theoretical values are those obtained for $c w=C W_{\min }$, as in the idealized 802.11 the absence of collisions means that the contention window is never doubled.
} 


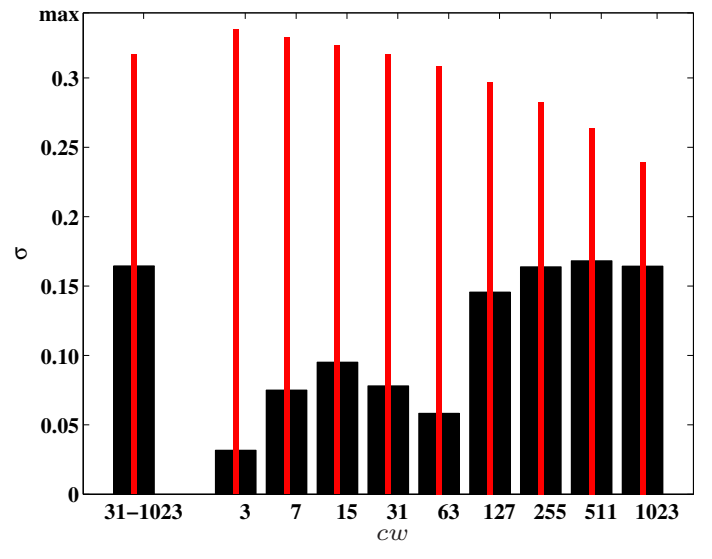

(a) Average spatial reuse

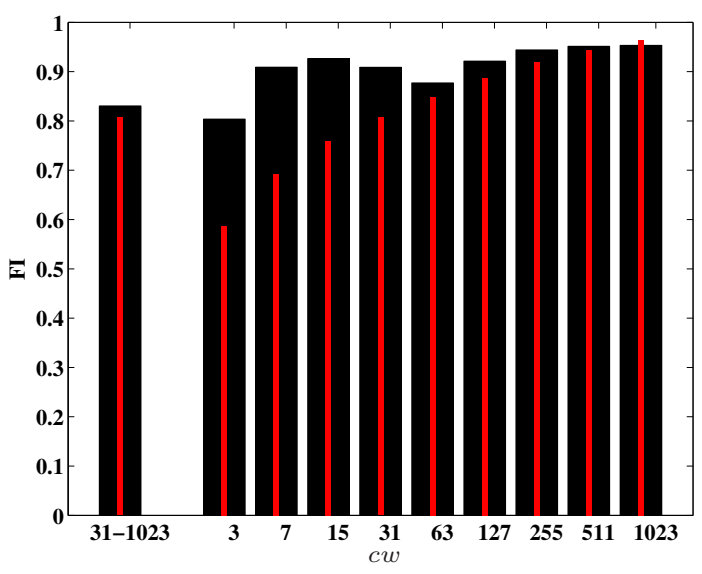

(b) Fairness index (long-term)

Fig. 5. CSRange $=$ RXRange $=250 \mathrm{~m}$ (1 space unit). The theoretical values (idealized 802.11 model) are in gray (thin bars), the values for (ns2) IEEE 802.11 are in black. The left-most bar corresponds to the variable contention window case (i.e., $C W_{\min }=31$ and $C W_{\max }=1023$ ), the other bars correspond to fixed contention window values (i.e., $c w=C W_{\min }=$ $\left.C W_{\max }\right)$. The plots show the average spatial reuse $(\sigma)$ and the fairness index (FI) of the protocol. The $95 \%$ confidence intervals are not represented on the figure as their small (below \pm 0.001 ) values make them barely visible.

$(c w=1023)$. Looking more closely at the trade-off between spatial-reuse and fairness, we observe that given a minimum fairness requirement the idealized protocol can guarantee a higher level of spatial reuse than IEEE 802.11. For example, at a FI requirement of 0.95 , the idealized protocol can guarantee $\sigma=0.24$, whereas IEEE 802.11 only achieves $\sigma=0.17$. The difference is even more striking at lower FI requirements.

In [12], we identified three factors that are responsible for this huge gap of performance, and which are

- the gagged node. A node is silenced by repeated Request-To-Send (RTS) messages that are not followed by a data packet transmission;

- the jammed node. A node is jammed by a data packet transmitted between two other nodes and cannot extract valuable state information in control messages sent concurrently;

- the focused node. A node focuses its transmission at- tempts on a single of its neighboring node that experiences high contention.

These three inefficiencies are studied in depth in [12], where a remedy is proposed to address each of them. In the variable contention window case, the suppression of these three situations brings the spatial reuse of IEEE 802.11 within $5 \%$ $($ CSRange $=250 \mathrm{~m})$ to $15 \%($ CSRange $=550 \mathrm{~m})$ of the spatial reuse of the idealized protocol. As a proof of concept, we reduce the size of the overhead control messages (RTS, CTS, and ACK) and check that the performance of the protocol converges to the theoretical values.

Figure 6 shows the performance of the modified IEEE 802.11 protocol when the overhead has been significantly reduced; we divided the time needed to send the control packets by 32 . We observe that the spatial reuse of the protocol converges to the theoretical limit at all values of $c w$. The small gap that remains at low $c w$ values is due to the remaining overhead and to the discrete nature of the backoff distribution, which makes it impossible to completely avoid collisions. Table I shows that the fairness of the protocol also converges to the theoretical values. The few unavoidable collisions at low fixed $c w$ slightly improve the fairness of the protocol compared to the theoretical prediction but it decreases its fairness in the variable contention window case. It is legitimate to ask if a sole reduction of the overhead in the current protocol would not bring equally good performances. This is however not the case, if we apply the same reduction of overhead to the current protocol with variable contention window $c w \in[31,1023]$, we obtain a spatial reuse $40 \%$ lower than with our modified protocol. At $c w=31$, where the gagged node situation is a serious problem, and a reduction of the overhead in the idealized protocol has a devastating effect on the spatial reuse, which basically drops to zero. In general, the gagged, jammed, and focused node situation appear independently of the time needed to send the RTS-CTS packets. It is therefore important to solve these situations if IEEE 802.11 is to benefit from a reduction of its overhead.

\section{NON SATURATED NETWORK}

We consider again a network on a line, with the same communication range and nearest neighbor intereference (see Figure 7) as in Section II. However, instead of having all nodes saturated, now only one node at one end of the network (the source, Node 0) is saturated, all the other nodes (the relays 1 to $N-1$ and the destination $N$ ) do not inject any new traffic in the network. Another difference is that RST/CTS messages may turned off or on, and we will see in Sections III-B and III-C the consequences of this option on the network stability. A common scenario in these networks occurs when the gateway of a wireless mesh network (WMN) needs to send packets to an end-user that is beyond its direct coverage range. To achieve this goal, the packets transit through the wireless backbone, forwarded by multiple relay nodes.

In this setting, measurements on a testbed deployed at EPFL show a rather unexpected behavior of 802.11 multihop networks, which is the striking difference in stability between 


\begin{tabular}{|c|c|c|c|c|c|c|}
\hline$c w$ & $\begin{array}{c}\text { IEEE } \\
802.11\end{array}$ & $\begin{array}{c}\text { gagged } \\
\text { solved }\end{array}$ & $\begin{array}{c}\text { jammed } \\
\text { solved }\end{array}$ & $\begin{array}{c}\text { focused } \\
\text { solved }\end{array}$ & $\begin{array}{c}\text { overhead } \\
\text { reduced }\end{array}$ & $\begin{array}{c}\text { idealized } \\
802.11\end{array}$ \\
\hline $31-1023$ & 0.83 & $0.73 \downarrow$ & $0.81 \uparrow$ & $0.45 \downarrow$ & 0.62 & 0.81 \\
\hline 3 & 0.80 & $0.73 \downarrow$ & $0.75 \simeq$ & $0.83 \uparrow$ & 0.69 & 0.59 \\
\hline 7 & 0.91 & $0.77 \downarrow$ & $0.78 \simeq$ & $0.82 \simeq$ & 0.73 & 0.69 \\
\hline 15 & 0.93 & $0.82 \downarrow$ & $0.81 \simeq$ & $0.82 \simeq$ & 0.76 & 0.76 \\
\hline 31 & 0.91 & $0.85 \downarrow$ & $0.85 \simeq$ & $0.82 \simeq$ & 0.81 & 0.81 \\
\hline 63 & 0.88 & $0.87 \simeq$ & $0.88 \simeq$ & $0.84 \simeq$ & 0.85 & 0.85 \\
\hline 127 & 0.92 & $0.89 \simeq$ & $0.90 \simeq$ & $0.87 \simeq$ & 0.89 & 0.89 \\
\hline 255 & 0.94 & $0.91 \simeq$ & $0.91 \simeq$ & $0.91 \simeq$ & 0.92 & 0.92 \\
\hline 511 & 0.95 & $0.92 \simeq$ & $0.93 \simeq$ & $0.94 \simeq$ & 0.94 & 0.94 \\
\hline 1023 & 0.95 & $0.94 \simeq$ & $0.94 \simeq$ & $0.96 \simeq$ & 0.96 & 0.96 \\
\hline
\end{tabular}

TABLE I

FAIRNESS INDEX OF IEEE 802.11 FOR CSRANGE $=$ RXRANGE $=250 \mathrm{M}$. WE USE THE SYMBOL $\simeq$ IF THE VARIATION IN THE VALUE OF THE FAIRNESS INDEX BETWEEN TWO CONSECUTIVE COLUMNS IS LESS THAN 0.05.

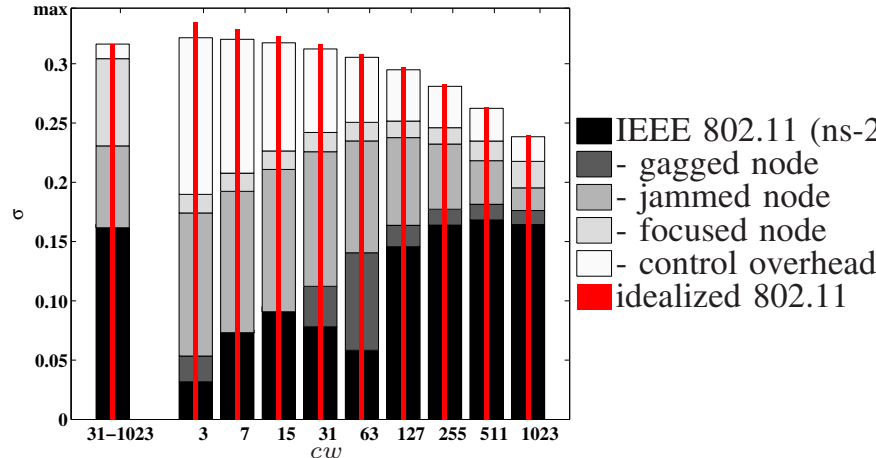

Fig. 6. Spatial reuse of IEEE 802.11 (CSRange $=$ RXRange $=250 \mathrm{~m}$ ). Legend. The theoretical values (idealized 802.11 protocol) are represented by the thin dark gray bars. The values for the current (ns-2) IEEE 802.11 protocol are in black (thick bars). We first solve the gagged node situation (dark gray) by using a reduced NAV on RTS. We then solve the jammed node situation (gray) by using an additional control channel, and the focused node situation (light gray) by using a backoff per link. Finally, as a proof of concept we reduce the size of the overhead (white).

3- and 4-hop networks. Our testbed is composed of two laptops connected to a router that act as the source (Node 0) and destination (Node $N=4$ ) of the traffic and three other wireless routers that act as relays (Nodes 1, 2 and 3). Each laptop runs on Linux with the software Iperf $^{2}$ used to generate saturated UDP traffic with payload size of 1470 bytes. Each

${ }^{2}$ Iperf - The TCP/UDP bandwidth measurement tool:
http://dast.nlanr.net/Projects/Iperf/

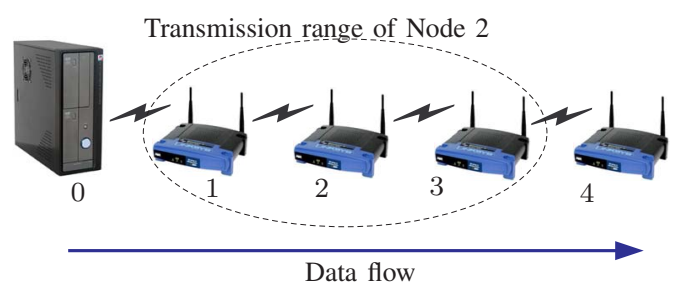

Fig. 7. Linear topology of a WMN backbone: Node 0 is the source (Wireless Access Point), Nodes 1, 2 and 3 are the relays (Transit Access Points) and Node 4 is the destination laptop is then connected through a wired cable to either node 0 or node 4 . The wireless routers are Asus WL-500gP running the version Kamikaze 7.07 of the OpenWRT firmware ${ }^{3}$. We change the mini-PCI WiFi cards to Atheros cards in order to benefit from the flexibility of the MadWifi driver [13]. We then set the routers to run in ad-hoc mode on channel 13 of IEEE $802.11 \mathrm{~b}$ at the data rate of $1 \mathrm{Mb} / \mathrm{s}$ and without RTS/CTS. To avoid interference from neighboring networks, we perform our measurements in the basement of the BC building at EPFL, where no other wireless network could be sensed. In this testbed, direct neighbors can communicate together but nodes separated by two hops or more cannot hear each other, as described earlier.

The difference between the behaviors of the 3-hop and 4hop topologies is clear. Figure 8 shows that the 3-hop topology is stable, but the 4-hop network is unstable. Furthermore, the 4-hop instability is due to node 1 , whose queue length indefinitely grows until it reaches the hardware limit (50 packets for our routers).

The problem that we tackle in this section is to better understand the root causes behind this experimental stability result. Toward this goal, we introduce an analytical model that is inspired from the behavior of CSMA/CA protocols (e.g., 802.11-like protocols) with some necessary simplifications for the sake of tractability.

\section{A. Discrete Markov Chain Model}

We now mathematically formalize the model [3]. All packets are generated by Node 0 , and are forwarded to the last node $N$ by successive transmissions via the intermediate nodes 1 to $N-1$. A time step $n \in \mathbb{N}$ corresponds to the successful transmission of a packet from some node $i$ to its neighbor $i+1$, or if $N$ is large enough, of a set of packets from different non-interfering nodes $i, j, \ldots$ to nodes $i+1, j+1, \ldots$, provided these transmissions overlap in time (the transmitters and receivers must therefore not interfere with each other). Remember that node 0 always has packets to transmit (infinite queue), and that the destination $N$ consumes immediately the packets, hence its queue length is always 0 . We are interested in the evolution of the queue sizes $b_{i}$ of the relaying nodes

\footnotetext{
${ }^{3}$ OpenWRT firmware: http://openwrt.org/
} 
3-hop

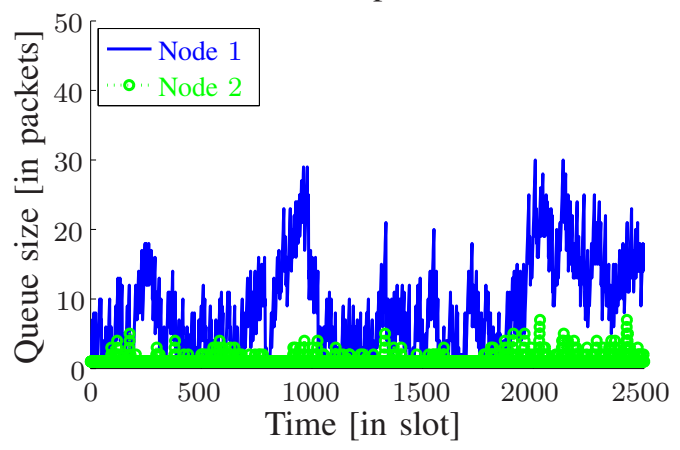

4-hop

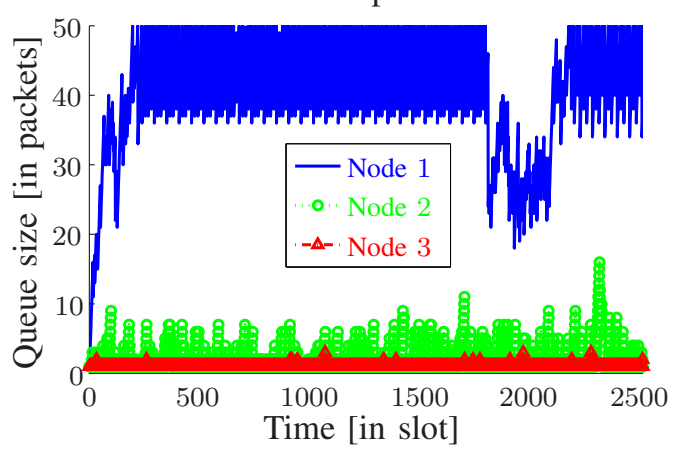

Fig. 8. Experimental results for the queue evolution of each relay node in 3- and 4-hop topologies. A time slot corresponds to an event when the buffer size is recorded, that is, every time a packet arrives at a node. A plot of the queue size versus time in seconds is given in the two first figures in Figure 12.

$1 \leq i \leq N-1$ over time, and therefore we adopt as a state variable of the system at time $n$ the vector

$$
\vec{b}(n)=\left[b_{1}(n) b_{2}(n) \ldots b_{N-1}(n)\right]^{T},
$$

with $T$ denoting transposition. We also introduce a set of $N$ auxiliary binary variables $z_{i}, 0 \leq i \leq N-1$, representing the $i^{t h}$ link activity at time slot $n: z_{i}(n)=1$ if a packet was successfully transmitted from node $i$ to node $i+1$ during the $n^{\text {th }}$ time slot, and $z_{i}(n)=0$ otherwise. Observing that

$$
b_{i}(n+1)=b_{i}(n)+z_{i-1}(n)-z_{i}(n),
$$

we can recast the dynamics of the system as

$$
\vec{b}(n+1)=\vec{b}(n)+A * \vec{z}(n)
$$

where

$$
\begin{aligned}
\vec{z}(n)= & {\left[z_{0}(n) z_{1}(n) z_{2}(n) \ldots z_{N-1}(n)\right]^{T} } \\
A= & {\left[\begin{array}{ccccc}
1 & -1 & 0 & \ldots & 0 \\
0 & 1 & -1 & 0 & \vdots \\
\vdots & \ddots & \ddots & \ddots & 0 \\
0 & \ldots & 0 & 1 & -1
\end{array}\right] }
\end{aligned}
$$

Finally, the activity of a link $z_{i}$ depends on the queue sizes of all the nodes, which we cast as $z_{i}=g_{i}(\vec{b})$ for some random function $g_{i}(\cdot)$ of the queue size vector, or in vector form as

$$
\vec{z}(n)=g(\vec{b}(n)) .
$$

The specification of $g=\left[g_{0}, \ldots, g_{N-1}\right]^{T}$ is the less straightforward part of the model, as it requires to enter in some additional details of the CSMA/CA protocol, which we defer to the next sections. We will detail it in Section III-C for a $N=3$ hops network. For larger networks, the specification of $g$ comes with some level of complexity, see [3]. Nevertheless, we can already mention here two simple constraints that $g$ must verify:

1) node $i$ cannot transmit if its buffer is empty, and therefore we have $z_{i}=g_{i}(\vec{b})=0$ if $b_{i}=0$;

2) nodes that successfully transmit in the same time slot must be at least 2 hops apart, as otherwise the packet from node $i$ would collide at node $i+1$ with the packet from node $i+2$. Hence

$$
z_{i} z_{i+k}=0 \text { for } k \in\{-2,-1,1,2\} .
$$

We observe that (6) and (7) make the model a discrete-time, irreducible Markov chain. The (in)stability of the network coincides with its (non-)ergodicity.

\section{B. Stealing Effect}

The "stealing" effect [3] is a result of the well-known hidden node problem occurring only in multihop topologies, as shown by the example illustrated in Figure 9. When node $i$ first enters the link competition phase, node $i+2$ may be unaware of this transmission attempt. Because it senses the medium to be idle, node $i+2$ may therefore start a transmission to node $i+3$, even though it lost the competition phase because node $i+2$ selected a larger backoff than node $i$. As a result, a collision occurs at node $i+1$ because of the broadcast nature of the wireless medium. Node $i$ will therefore experience an unsuccessful transmission, whereas the transmission from node $i+2$ will succeed. We refer to this artifact as the stealing effect. It differs from the classical capture effect, which pertains to packets transmitted to the same destination. The stealing effect is the successful capture of the channel of a node from an upstream node that had seized the medium earlier. We define $p$ to be the probability of its occurrence.

In IEEE 802.11, the stealing effect corresponds to the event where node $i+2$ captures the channel, even though it has a larger backoff value than node $i$. The probability $p$ of this event depends on the specific protocol implementation. If the optional RTS/CTS handshake is disabled, then $p \rightarrow 1$. If RTS/CTS is enabled, then $p$ is typically much smaller, but

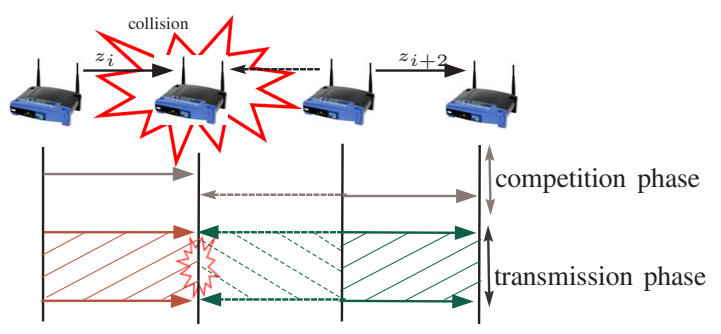

Fig. 9. Stealing effect scenario. 
still non-zero because RTS messages may collide [14]. Indeed, the transmission time of a control message (e.g., the RTS transmission time at the $1 \mathrm{Mb} / \mathrm{s}$ basic rate is $352 \mu \mathrm{s}$ ) is nonnegligible compared to the duration of a backoff slot $(20 \mu \mathrm{s})$. It is only in the equivalent idealized protocol of Section II-A that the instantaneous RTS/CTS exchange would amount to take $p=0$.

In our model, the stealing effect is captured by having the function $g(\cdot)$ in (7) depend on $p$. As we will see in Section III-C, a positive and somewhat counterintuitive consequence of the stealing effect is the smooth propagation of packets along the linear network. Indeed, by favoring downstream links over upstream ones, a form of virtual back-pressure is enabled, which prevents packets from being injected too rapidly by the source into the network.

\section{Stability}

Let us first analyze the 3-hop topology, which remains relatively simple because only one link can be active at a given time slot. Indeed, the only three possible transmission patterns are

$$
\vec{z} \in\left\{\left[\begin{array}{lll}
1 & 0 & 0
\end{array}\right]^{T},\left[\begin{array}{lll}
0 & 1 & 0
\end{array}\right]^{T},\left[\begin{array}{lll}
0 & 0 & 1
\end{array}\right]^{T}\right\} .
$$

We can now complete the description of the function $g(\cdot)$, before analyzing the ergodicity of the Markov chain. We say that a queue is stable when it does not tend to increase forever. A queue is stable when its evolution is ergodic (it goes back to zero almost surely in finite time). A network is stable when the queues of all forwarding nodes are stable.

The role of the stochastic function $g(\cdot)$ is to map a buffer status $\vec{b}$ to a transmission pattern $\vec{z}$ with a certain probability.

First, in the case of an idealized CSMA/CA model without the stealing effect $(p=0)$, all non-empty nodes have exactly the same probability of being scheduled. That is, if only node 0 and node 1 (or, respectively, node 2 ) have a packet to send, both patterns $\left[\begin{array}{lll}1 & 0 & 0\end{array}\right]^{T}$ and $\left[\begin{array}{lll}0 & 1 & 0\end{array}\right]^{T}$ (resp., $\left[\begin{array}{lll}0 & 0 & 1\end{array}\right]^{T}$ ) occur with probability $1 / 2$. Similarly, when all three nodes have a packet to send, each of the three possible transmission patterns happens with probability $1 / 3$.

More generally, when we include the stealing effect, we capture the bias towards downstream links that are two hops away. When only node 0 and node 1 compete for the channel, nothing is changed and the probability of success remains $1 / 2$ as they are only separated by one single hop. However, when node 0 and node 2 compete together, there is a probability $p$ that node 2 steals the channel.

This leads us to define function $g(\cdot)$ differently for each region of $\mathbb{N}^{2}$ as shown in Figure 10. First, in region $A=$ $\left\{b_{1}(n)=0, b_{2}(n)=0\right\}$,

$$
g\left(\left[b_{1}(n) b_{2}(n)\right]^{T}\right)=\left[\begin{array}{lll}
1 & 0 & 0
\end{array}\right]^{T} .
$$

In region $B=\left\{b_{1}(n)>0, b_{2}(n)=0\right\}$ we have that

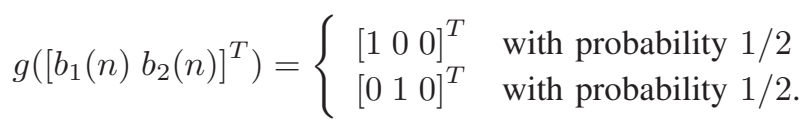

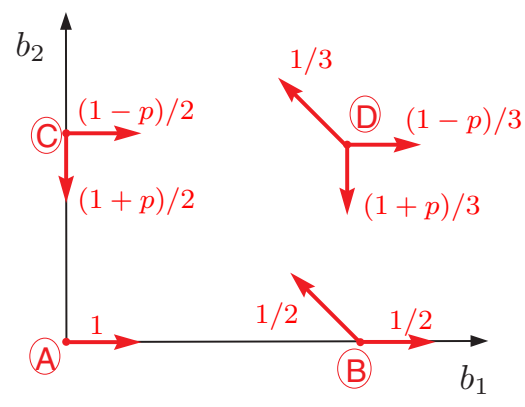

Fig. 10. Random walk in $\mathbb{N}^{2}$ modeling the 3-hop network. where the 4 regions are: (A) $\{0 ; 0\}$, (B) $\left\{b_{1}>0 ; 0\right\}$, (C) $\left\{0 ; b_{2}>0\right\}$ and (D) $\left\{b_{1}>0 ; b_{2}>0\right\}$.

In region $C=\left\{b_{1}(n)=0, b_{2}(n)>0\right\}$,

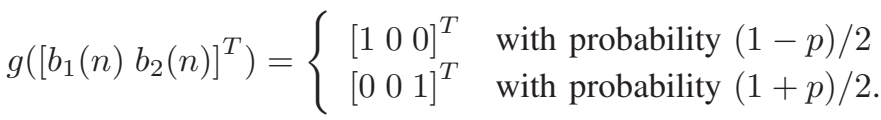

Finally, in region $D=\left\{b_{1}(n)>0, b_{2}(n)>0\right\}$, all three nodes compete, and node 2 can still steal the channel from node 0 , hence

$g\left(\left[b_{1}(n) b_{2}(n)\right]^{T}\right)= \begin{cases}{\left[\begin{array}{lll}1 & 0 & 0\end{array}\right]^{T}} & \text { with probability }(1-p) / 3 \\ {\left[\begin{array}{lll}0 & 1 & 0\end{array}\right]^{T}} & \text { with probability } 1 / 3 \\ {\left[\begin{array}{lll}0 & 0 & 1\end{array}\right]^{T}} & \text { with probability }(1+p) / 3 .\end{cases}$

The queue evolution from (6) is a random walk in $\mathbb{N}^{2}$, and its ergodicity can be easily analyzed using Foster-Lyapunov techniques. Theorem 1 shows the stabilizing influence of the stealing effect, for the 3-hop network.

A similar random walk in $\mathbb{N}^{3}$ models the 4-hop network, which can be shown now to be unstable, whether the stealing effect occurs or not. The following theorem, which is proven in [3], summarizes the results.

Theorem 1: A 3-hop network is stable if and only if $0<$ $p \leq 1$. A 4-hop network is unstable for all $0 \leq p \leq 1$.

\section{Experimental Validation and Instability problem at higher rates}

The Markovian model explains why a 3-hop network is stable, but becomes unstable when a $4^{\text {th }}$ hop is added (see Figure 8). Nevertheless, the results from Figure 8 are obtained with a fixed data rate of $1 \mathrm{Mb} / \mathrm{s}$, a buffer size limit of

\begin{tabular}{|c|c|c|c|c|}
\hline throughput $\backslash$ rate & $1 \mathrm{Mb}$ & $2 \mathrm{Mb}$ & $11 \mathrm{Mb}$ & auto-rate \\
\hline 11 & $894 \mathrm{~kb} / \mathrm{s}$ & $1.67 \mathrm{Mb} / \mathrm{s}$ & $6.71 \mathrm{Mb} / \mathrm{s}$ & $5.79 \mathrm{Mb} / \mathrm{s}$ \\
12 & $858 \mathrm{~kb} / \mathrm{s}$ & $1.52 \mathrm{Mkb} / \mathrm{s}$ & $5.82 \mathrm{Mb} / \mathrm{s}$ & $2.03 \mathrm{Mb} / \mathrm{s}$ \\
13 & $754 \mathrm{~kb} / \mathrm{s}$ & $1.28 \mathrm{Mb} / \mathrm{s}$ & $4.23 \mathrm{Mb} / \mathrm{s}$ & $1.95 \mathrm{Mb} / \mathrm{s}$ \\
14 & $813 \mathrm{~kb} / \mathrm{s}$ & $1.6 \mathrm{Mkb} / \mathrm{s}$ & $5.98 \mathrm{Mb} / \mathrm{s}$ & $5.49 \mathrm{Mb} / \mathrm{s}$ \\
\hline 3-hop & $241 \mathrm{~kb} / \mathrm{s}$ & $493 \mathrm{~kb} / \mathrm{s}$ & $1.05 \mathrm{Mb} / \mathrm{s}$ & $373 \mathrm{~kb} / \mathrm{s}$ \\
\hline 4-hop & $194 \mathrm{~kb} / \mathrm{s}$ & $354 \mathrm{~kb} / \mathrm{s}$ & $791 \mathrm{~kb} / \mathrm{s}$ & $260 \mathrm{~kb} / \mathrm{s}$ \\
\hline
\end{tabular}

TABLE II

MEASUREMENTS OF THE LINKS THROUGHPUT AND THE END-TO-END THROUGHPUT OF A 3- AND 4-HOP LINEAR TOPOLOGY FOR DIFFERENT DATA RATES. 
50 packets, and a small-scale testbed where the routers are used without their external antennas (better control on the experimental environment). In order to validate our results on a different setting, we modify the MadWifi driver to unlock the buffer size limit and to allow the modification of its value at run time through simple commands. We then set the buffer limit to 100 packets and repeat the experiment from Figure 8 on the real-scale deployment of Figure 11, for different data rate settings.

Figures 12 and 13 show the queue evolution of a 3-hop network (nodes 0 to 3 in Figure 11) and a 4-hop network (nodes 0 to 4 in Figure 11) at data rates of: $1 \mathrm{Mb} / \mathrm{s}, 2 \mathrm{Mb} / \mathrm{s}$, $11 \mathrm{Mb} / \mathrm{s}$ and auto-rate. Additionally, Table II presents the link throughputs and the end-to-end throughputs achieved at the different data rates.

Our results show that even though $l_{3}=(2,3)$ is the bottleneck link for all the data rates, it does not result in making the 3-hop network unstable, because of the stealing effect described in Section III-B. Moreover, the simple addition of a $4^{\text {th }}$ hop turns the network from being stable to becoming unstable (i.e., the queue remains close to the buffer limit). We note that the queue size variations are larger than in Figure 8. This is explained by the lack of control in the realscale deployment, which is more prone to changing channel conditions. Nevertheless, we stress that, despite the variations, the change in stability between a 3-and 4-hop network is seen for all the different data rates that we tested, as predicted by the analytical model of Section III-C.

Our experimental results at higher rates also provided an interesting observation that is worth mentioning. Indeed, when looking at the 3-hop results, we see that the queue size of node 1 increases at higher rate, both in mean and variance. To understand why, remember that the transmission duration decreases at larger rates. As a result, the period of vulnerability to the stealing effect decreases at larger rates as well. Therefore the probability $p$ of stealing effect decreases as a function of the data transmission rate.

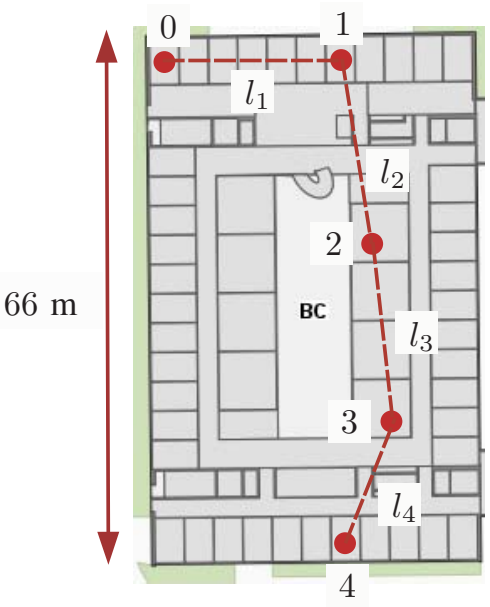

Fig. 11. Map of the testbed deployment used in Section III-D.
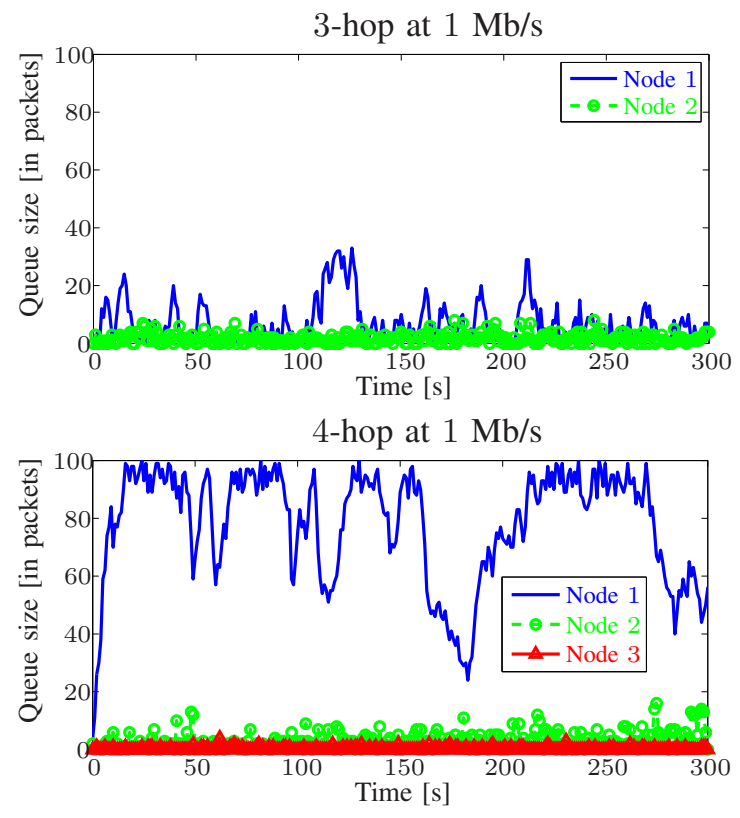

3-hop at $2 \mathrm{Mb} / \mathrm{s}$

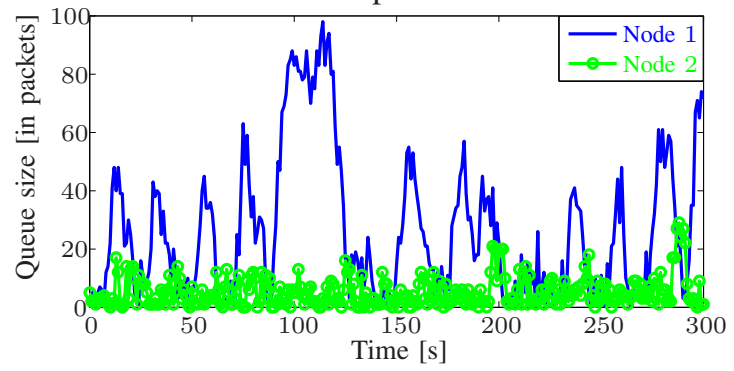

4-hop at $2 \mathrm{Mb} / \mathrm{s}$

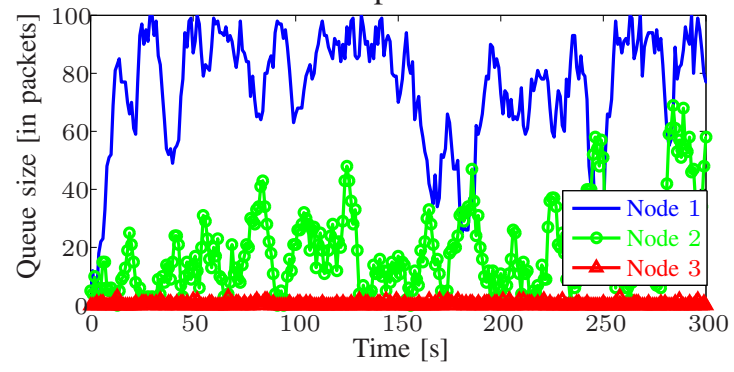

Fig. 12. Validation of the experimental results from Figure 8 on a different setup running at $1 \mathrm{Mb} / \mathrm{s}$ and $2 \mathrm{Mb} / \mathrm{s}$, for 3 and 4 hops.

As we have seen in Section III-C, the smaller $p$, the more the queue of Node 1 grows. This is indeed what we observe in Figures 12 and 13. Our experimental results at higher rates confirm the predictions made from the analytical model described in Section III-C.

\section{RELATED STATE OF THE ART}

A considerable amount of work has been devoted in the recent year to the study of decentralized CSMA schemes that do not require message passing from other nodes in multi-hop networks.

In the saturated case, the loss network model [4] was first proposed by $[15,16]$ to model the CSMA protocol and later 
3-hop at $11 \mathrm{Mb} / \mathrm{s}$

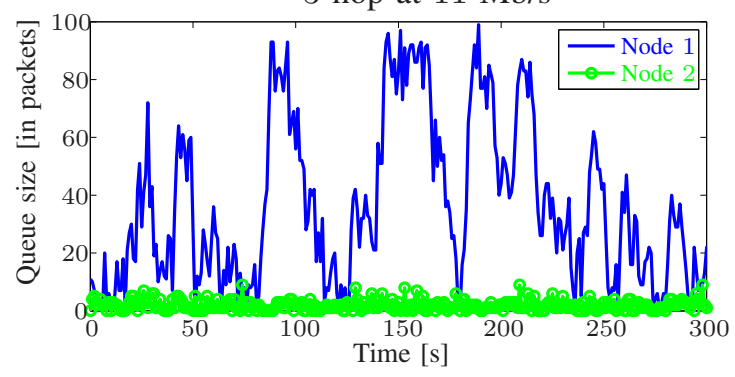

4-hop at $11 \mathrm{Mb} / \mathrm{s}$

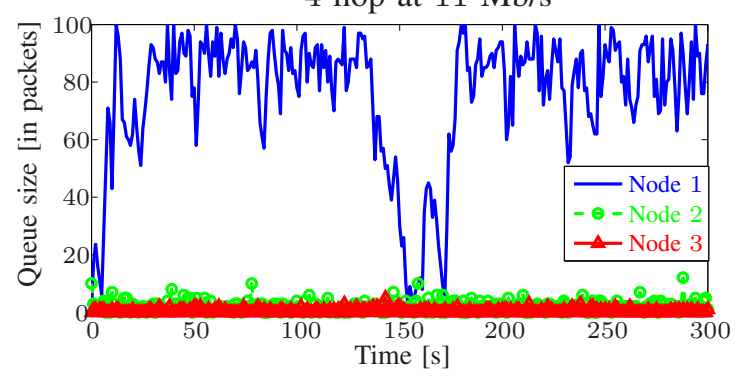

3-hop with auto-rate

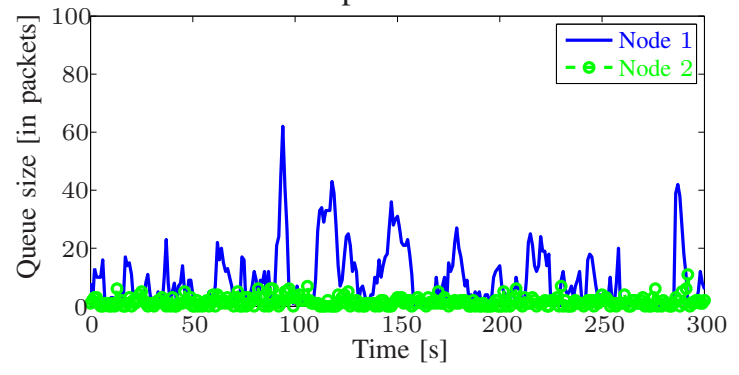

4-hop with auto-rate

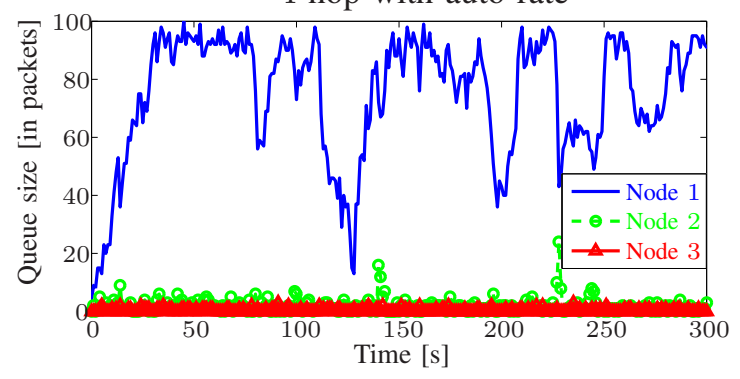

Fig. 13. Validation of the experimental results from Figure 8 on a different setup running at $11 \mathrm{Mb} / \mathrm{s}$ and with autorate configuraiton.

extended to an idealized CSMA/CA protocol by [5] and [6]. The strength of this model is to preserve the dependence between nodes, which is typical of CSMA protocols. Using this model, which is also known as the hard core model in statistical physics, [5] is probably the first to predict and explain the starvation phenomenon in multi-hop topologies. [6] extends the results of [5] and provides a closed form expression for the spatial reuse of CSMA/CA protocols in large one-dimensional networks. [1] extends the results further to consider the effect of the receiving and carrier sensing ranges on the model. [17] extends the model to allow for nodedependent backoff parameters, and show how to compute them to remove the unfairness completely in 1-dim linear networks.
In the non-saturated case, many schemes have been theoretically proven to be throughput-optimal or almost throughput optimal [18-21]. Despite this significant body of analytical work, almost all the existing solutions are still quite far from being compatible with the current IEEE 802.11 protocol, and require in general to know the feasible capacity region. A possible solution is to estimate it before running the MAC algorithm and to then use an optimization-based rate control at the network layer [22]. Another practical scheme to circumvent the instability problem described in Section III-C is the hop-by-hop congestion control mechanism DiffQ in [23], which is a protocol implementing a form of backpressure (i.e., prioritizing links with large backlog differential). To achieve this implementation, DiffQ lets each node inform its neighbors of its queue size by piggybacking this information in the data packet. EZ-flow [24] does not require to modify the packet structure, and exploits instead the broadcast nature of the wireless channel to estimate the queue size of the next downstream node, instead of computing the differential backlog between the queue sizes of two consecutive nodes, which push back an implicit congestion indication more rapidly to the source. Finally, stationary large buffer probabilities of the 3-hop model of Section III-A, which capture the effect of $p$, have been recently obtained in [25].

Other recent models of CSMA/CA networks include [26], [9], [27]. These models incorporate more features of the real protocols than the two models described in this paper and typically lead to large systems of equations that must be numerically solved. These models offer therefore more limited insight into the behavior of CSMA/CA protocols in large multi-hop networks. However, some of these works do observe the starvation phenomenon. In particular, [9] attributes the unfairness of CSMA/CA protocols to a coordination problem inherent to carrier sensing protocols. [27] reports more optimistic results about the fairness of 802.11, but this paper assumes that flows are rate-controlled at the source, whereas we do not make such an assumption. [28] studies a variant of CSMA/CA protocol for bufferless relay nodes.

\section{CONCLUSION}

We have presented two models of wireless multihop networks. They are in general simpler than most models proposed so far. When confronting them with realistic simulations or experiments, we find that they still capture the essential performance properties found in the real protocol. It is worth noting that in both cases the imperfections of the real protocol (collisions, stealing effect) help us to mitigate starvation or instability. The explanation follows from the models described in the paper.

The loss network model is well suited to study the performance of saturated networks. It explains in particular why CSMA/CA networks achieve a very high spatial reuse, but also suffer from an intrinsic unfairness at the link level already. We have also described the main difference between the model and the real 802.11 network protocol. 
Non saturated networks are much more difficult to model. We have proposed a discrete-time Markovian model, which works well as long as the network diameter remains small enough. Interestingly enough, the change of behavior occurs when this diameter moves from 3 to 4 hops, and we showed this transition both theoretically on the model and experimentally in the testbed.

\section{ACKNOWLEDGMENT}

The work presented in this paper was supported (in part) by the National Competence Center in Research on Mobile Information and Communication Systems (NCCR-MICS), a center supported by the Swiss National Science Foundation under grant number 5005-67322.

\section{REFERENCES}

[1] M. Durvy, O. Dousse, and P. Thiran, "Self-Organization Properties of CSMA/CA Systems and Their Consequences on Fairness," IEEE Transactions on Information Theory, vol. 55, no. 3, pp. 931-943, 2009.

[2] __, "On the Fairness of Large CSMA Networks," IEEE Journal on Selected Areas in Communications, vol. 27, no. 7, pp. 1093-1104, 2009.

[3] A. Aziz, D. Starobinski, and P. Thiran, "Elucidating the instability of random access wireless mesh networks," in Proc. of SECON, Rome, Italy, June 2009.

[4] F. P. Kelly, "Loss networks," Ann. Appl. Probab., vol. 1, no. 3, pp. 319 378, 1991.

[5] X. Wang and K. Kar, "Throughput Modelling and Fairness Issues in CSMA/CA Based Ad-Hoc Networks," in INFOCOM, Miami, 2005.

[6] M. Durvy and P. Thiran, "A Packing Approach to Compare Slotted and Non-Slotted Medium Access Control," in INFOCOM, Barcelona, 2006.

[7] Y. Baryshnikov, E. G. J. Coffman, and P. Jelenkovic, "Space filling and depletion," J. Appl. Probab., vol. 42, pp. 691-702, 2004.

[8] R. Jain, The Art of Computer Systems Performance Analysis. John Wiley and Sons, Inc., 1991.

[9] M. Garetto, T. Salonidis, and E. Knightly, "Modeling Per-flow Throughput And Capturing Starvation in CSMA Multi-hop Wireless Networks," IEEE/ACM Transctions on Networking, vol. 16, no. 4, pp. 864-877, 2008.

[10] C. Chaudet, D. Dhoutaut, and I. G. Lassous, "Experiments of Some Performance Issues with IEEE 802.11b in Ad Hoc Networks," in WONS, St Moritz, 2005.

[11] "Network simulator 2 (ns-2)," http://www.isi.edu/nsnam/ns/.

[12] M. Durvy and P. Thiran, "Understanding the Gap between the IEEE 802.11 Protocol Performance and the Theoretical Limits," in SECON, Reston, 2006.

[13] The Madwifi project, http://madwifi-project.org/.

[14] J. Shi, O. Gurewitz, V. Mancuso, J. Camp, and E. Knightly, "Measurement and modeling of the origins of starvation in congestion controlled mesh networks," in Proc. of INFOCOM, Phoenix, AZ, Apr. 2008.

[15] F. A. Tobagi, "Modeling and performance analysis of multihop packet radio networks," Proc. IEEE, vol. 75, no. 1, pp. 135-155, 1987.

[16] R. Boorstyn, A. Kershenbaum, B. Maglaris, and V. Sahin, "Throughput analysis in multihop CSMA packet radio networks," IEEE Trans. Commun., vol. 35, no. 3, pp. 267-274, 1987.

[17] P. van de Ven, J. van Leeuwaarden, D. Denteneer, and A. Janssen, "Spatial fairness in linear random-access networks," Performance Evaluation, vol. 67, pp. 1230-1242, 2010.

[18] A. Proutière, Y. Yi, and M. Chiang, "Throughput of random access without message passing," in Proc. of CISS, Princeton, NJ, Mar. 2008.

[19] J. Shin, D. Shah, and S. Rajagopalan, "Network adiabatic theorem: An efficient randomized protocol for contention resolution," in Proc. of SIGMETRICS, Seattle, WA, June 2009.

[20] L. Jiang and J. Walrand, "A distributed CSMA for throughput and utility maximization in wireless networks," in Proc. Allerton, September 2008.

[21] P. Marbach, A. Eryilmaz, and A. Ozdaglar, "Achievable rate region of CSMA schedulers in wireless networks with primary interferences constraints," in Proc. IEEE Conf. on Decision and Control'07, December 2007.
[22] T. Salonidis, G. Sotiropoulos, R. Guerin, and R. Govindan, "Online optimization of 802.11 mesh networks," in Proc. of CoNEXT, Rome Italy, Dec. 2009.

[23] A. Warrier, S. Janakiraman, S. Ha, and I. Rhee, "Diffq: Practical differential backlog congestion control for wireless networks," in Proc. of INFOCOM, Rio de Janeiro, Brazil, 2009.

[24] A. Aziz, D. Starobinski, P. Thiran, and A. El Fawal, "Ez-flow: Removing turbulence in ieee 802.11 wireless mesh networks without message passing," in Proc. of CoNEXT, Rome, Italy, Dec. 2009.

[25] F. M. Guillemin, C. Knessl, and J. van Leeuwaarden, "Wireless multihop networks with stealing: large buffer asymptotics," in Proceedings ITC 2010, Amsterdam, September 2010.

[26] M. Garetto, J. Shi, and E. W. Knightly, "Modeling Media Access in Embedded Two-Flow Topologies of Multi-Hop Wireless Networks," in MobiCom, 2005.

[27] A. Jindal and K. Psounis, "The achievable rate region of 802.11scheduled multihop networks," IEEE/ACM Trans. Netw., vol. 17, no. 4 pp. 1118-1131, 2009.

[28] O. Dousse, "Revising buffering in CSMA/CA wireless multihop networks," in Proc. of SECON, San Diego, CA, June 2007. 